Please do not remove this page

RMIT

UNIVERSITY

\title{
Graphene-like nano-sheets/36deg LiTaO3 surface acoustic wave hydrogen gas sensor
}

Arsat, Rashidah; Breedon, Michael; Shafiei, Mahnaz; Kalantar Zadeh, Kourosh; Wlodarski, Wojciech; Gilje, Scott; Kaner, Richard

https://researchrepository.rmit.edu.au/esploro/outputs/9921863730301341/filesAndLinks?institution=61RMIT_INST\&index=null

Arsat, R., Breedon, M., Shafiei, M., Kalantar Zadeh, K., Wlodarski, W., Gilje, S., Kaner, R., \& Arregui, F. (2008). Graphene-like nano-sheets/36deg LiTaO3 surface acoustic wave hydrogen gas sensor.

Proceedings of the 7th IEEE Conference on Sensors, 188-191.

https://doi.org/10.1109/ICSENS.2008.4716414

Published Version: https://doi.org/10.1109/ICSENS.2008.4716414

Repository homepage: https://researchrepository.rmit.edu.au

(c) 2008 Crown. Personal use of this material is permitted. However, permission to reprint/republish this material for advertising or promotional purposes or for creating new collective works for resale or redistribution to servers or lists, or to reuse any copyrighted component of this work in other works must be obtained from the IEEE.

Downloaded On 2023/04/26 20:47:03 +1000 


\section{Graphene-like Nano-Sheets $/ 36^{\circ} \mathrm{LiTaO}_{3}$ Surface Acoustic Wave Hydrogen Gas Sensor}

\author{
Arsat R, Breedon M, Shafiei M, \\ Kalantar-zadeh K, Wlodarski W \\ RMIT University, City Campus, GPO Box 2476V \\ Melbourne 3001, Victoria, AUSTRALIA \\ Email: rashidah_arsat@ieee.org
}

\begin{abstract}
Presented is the material and gas sensing properties of graphene-like nano-sheets deposited on $36^{\circ} \mathrm{YX}$ lithium tantalate $\left(\mathrm{LiTaO}_{3}\right)$ surface acoustic wave (SAW) transducers. The graphene-like nano-sheets were characterized via scanning electron microscopy (SEM), atomic force microscopy (AFM) and X-ray photoelectron spectroscopy (XPS). The graphenelike nano-sheet/SAW sensors were exposed to different concentrations of hydrogen $\left(\mathrm{H}_{2}\right)$ gas in a synthetic air at room temperature. The developed sensors exhibit good sensitivity towards low concentrations of $\mathrm{H}_{2}$ in ambient conditions, as well as excellent dynamic performance towards $\mathrm{H}_{2}$ at room temperature.
\end{abstract}

\section{INTRODUCTION}

Interest in $\mathrm{H}_{2}$ gas sensing has enjoyed a skyrocketing popularity, as hydrogen has been touted as a panacea for clean energy production. It is highly flammable and explodes in air at concentrations as low as $4 \%$ [1]. Furthermore, $\mathrm{H}_{2}$ has a low ignition energy, posing a significant risk of deflagration. $\mathrm{H}_{2}$ sensing also finds important applications in aerospace, chemical refining and biomedical systems $[2,3]$.

A great deal of attention has been drawn to graphene due to its unique electrical and mechanical properties. As a monoatomically thick two-dimensional layer of $\mathrm{sp}^{2}$-bonded carbon, graphene can be considered as the basic building block of all graphitic materials, arranging itself into large planar sheets with repetitious benzene ring motif $[4,5]$.

To date, several synthesis methods have been developed by researchers in order to obtain a perfect single layer of graphene. Novoselov et al. [4-6] have micromechanically cleaved highly oriented pyrolytic graphite (HOPG) to obtain a single layer of graphene. The technique was repeated by Joshi et al. [7], who exfoliated the graphene onto doped silicon substrates. Furthermore, they [7] explored the potential of a single graphene layer for chemical sensing applications. Alternatively, graphene can be produced by the conversion of nanodiamond [8] and reduction of $\mathrm{SiC}$ [9], both of which are of high cost.

Authors thank Ministry of Higher Education, Malaysia and University of Technology Malaysia (UTM) for the financial support.

\author{
Gilje $S^{\mathrm{a}}$, Kaner R.B ${ }^{\mathrm{a}}$, Arregui F. J ${ }^{\mathrm{b}}$ \\ ${ }^{a}$ Dept of Chemistry and Biochemistry and California \\ Nanosystems Institute, UCLA, USA \\ ${ }^{\mathrm{b}}$ Public University of Navarre, Pamplona, Spain
}

Rapid progress for the electronic applications of graphene is anticipated. As graphene is an inexpensive lightweight material, it has been employed in room temperature operating transistors [10] and tunable storage media [11]. In addition, as it is a two-dimensional material, graphene has a large surface area with respect to volume. This large surface area should increase the interaction area available for hydrogen molecules, thus enhancing sensitivity. Schedin et al. [6] and Joshi et al. [7] have pioneered the investigation of graphene based chemical sensors. Joshi et al. [7] have fabricated and tested graphene based field effect transistors towards different gases. These have been supported by the experiment performed by Schedin et al. [6] to assess the effect of gaseous chemicals on graphene devices. Their Hall measurements have revealed that $\mathrm{NO}_{2}$ has acted as acceptor and $\mathrm{CO}$ as donor. Thus illustrating that upon exposure, the graphene surface has become p-type. Electrical baseline measurements and Raman spectroscopy $[6,7]$ confirm that analyte gases readily desorb from graphene based sensors.

In this paper, we have deposited graphene-like nanosheets on the active area of $36^{\circ} \mathrm{YX} \mathrm{LiTaO}_{3}$ SAW transducers and measured the frequency shifts towards different concentrations of $\mathrm{H}_{2}$ in dry synthetic air. To the best of authors' knowledge, sensing performance of SAW gas sensors based on graphene-like layer towards $\mathrm{H}_{2}$ is reported for the first time. The dynamic response of various concentrations of $\mathrm{H}_{2}$ gas at room operating temperature $\left(25^{\circ} \mathrm{C}\right)$, are presented in this paper.

\section{EXPERIMENTAL}

\section{A. Fabrication}

The SAW transducers were fabricated on a $36^{\circ} \mathrm{YX}$ $\mathrm{LiTaO}_{3}$ substrate using photolithography. It consists of 38 electrode pairs in input and output interdigitated transducers (IDTs) with a periodicity of $40 \mu \mathrm{m}$. These devices also have 160 reflectors in each side, and a $700 \mu \mathrm{m}$ aperture width. The center-to-center distance between the IDTs is $1920 \mu \mathrm{m}$. 
Metallization double layer of $\mathrm{Ti}(20 \mathrm{~nm})$ and $\mathrm{Au}(80 \mathrm{~nm})$ were patterned to form the device's IDTs and reflectors.

The graphene-like nano-sheets were chemically synthesized via the reduction of spray-coated graphite oxide (GO) with hydrazine [12]. GO was synthesized by Hummers method after which the GO was then dispersed in water and the spray-coated onto a preheated device. The as deposited GO device was placed in a flow cell and heated at $80^{\circ} \mathrm{C}$ under flowing helium gas. Hydrazine vapor was supplied via a bubbler to the system. The produced graphene layer was then characterized via SEM, AFM and XPS.

\section{B. Gas Sensing Measurement}

The sensor was placed in a computerized multi-channel gas calibration system. The system was used for generating 5 pulses of $\mathrm{H}_{2}$ gas $(0.06 \%, 0.125 \%, 0.25 \%, 0.50 \%$, and $1 \%)$ in a synthetic air. This sequence was utilized to expose the sensor at room operating temperature. The response to the $\mathrm{H}_{2}$ gas was monitored using a frequency counter (Fluke PM6680B) as change in frequency of the SAW sensor. The total flow rate of the gas was kept constant at $200 \mathrm{sccm}$, and synthetic air was used as the reference (baseline) gas and the carrier gas.

\section{RESULTS \& DISCUSSIONS}

SEM was conducted on graphene-like nano-sheets deposited onto SAW transducers. It can be seen in Fig. 1, that the graphene-like sheets (visible as pale grey regions) cover the area of the SAW device. Complementing this is in Fig. 2, is an AFM image which depicts numbers of graphene layers. It is estimated that the graphene-like sheets are monoatomically thick.

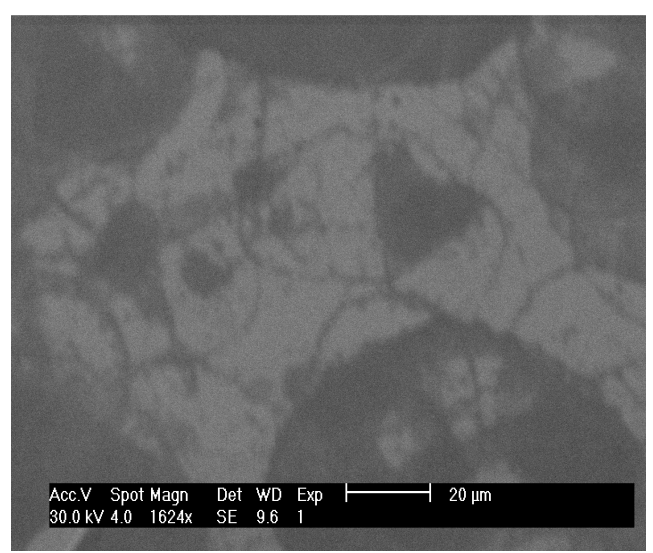

Figure 2: SEM image of graphene-like nano-sheets deposited on SAW transducers
Additionally, XPS (Fig. 3) has been provided. The C1s XPS spectrum has signified the existence of $\mathrm{C}-\mathrm{C}$ at $284.5 \mathrm{eV}$. Other carbon bonding schemes arising from different functional groups were observed in the spectrum. This is ascribed to the adventitious carbon present on the sample and the incomplete reduction of GO with hydrazine. It is believed that the incomplete reduction of $\mathrm{GO}$ with hydrazine results in the absence of the $\mathrm{C}=\mathrm{C}$ bond.

As such, we employed the nomenclature of graphenelike, rather than graphene. The deposited sensing layer displays graphene-like characteristics such as thickness, but XPS has concluded that the GO precursor has not been completely reduced to ideal, stoichometric graphene.

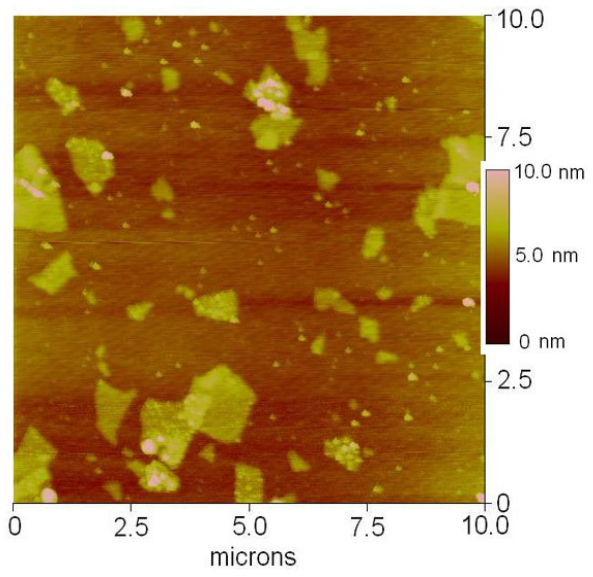

Figure 1: AFM image highlights the thickness of the graphene-like layers

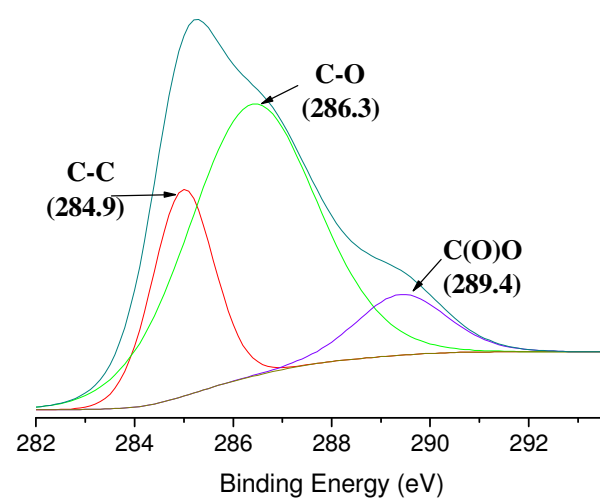

Figure 3: XPS analysis of graphene-like sheets 


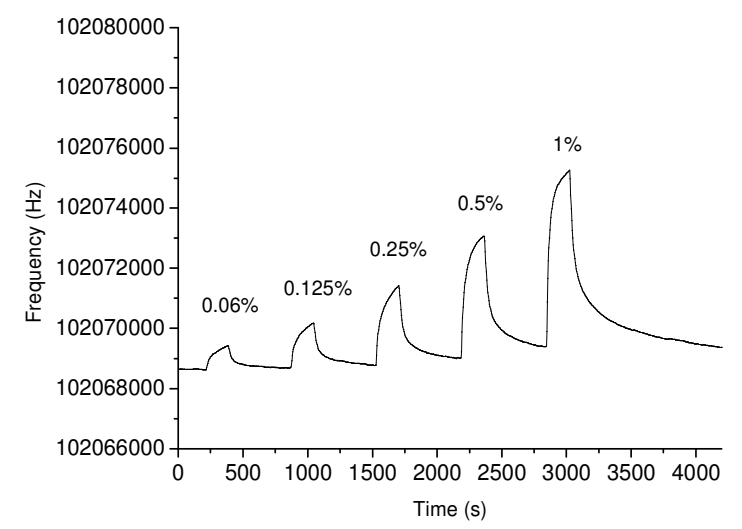

Figure 4: Response of graphene-like nano-sheets/SAW sensor towards $\mathrm{H}_{2}$ pulse sequence at room temperature

Fig. 4 shows the dynamic response of the graphene-like nano-sheets/SAW sensor to a sequence of different $\mathrm{H}_{2}$ gas concentrations in synthetic air at room temperature. The measured response at room temperature was $5.8 \mathrm{kHz}$ towards $1 \% \mathrm{H}_{2}$. This low temperature operation also is favorable in many applications, especially those involving low power operations and inflammable environment.

We also have measured the frequency response towards $\mathrm{NO}_{2}$ gas, and the frequency decreased upon exposure to $\mathrm{NO}_{2}$ gas and increased after the gas being purged out [13]. $\mathrm{NO}_{2}$ is an oxidizing gas whereas, $\mathrm{H}_{2}$ is one the important reducing gas. The opposite frequency responses show p-type response. This agrees with the report by Schedin et al. [6]

The frequency shifts of different $\mathrm{H}_{2}$ gas concentrations at room temperature are plotted in Fig. 5. It is observed that the frequency shift of $\mathrm{H}_{2}$ at this temperature increases almost linearly with the increase in $\mathrm{H}_{2}$ concentration.

The plotted graph in Fig. 6 shows the response and recovery time of measured SAW sensor towards $\mathrm{H}_{2}$ gas at room temperature. Exposed to $0.06 \% \mathrm{H}_{2}$, response and recovery time of $76 \mathrm{~s}$ and $128 \mathrm{~s}$ were observed, respectively. It is known that at elevated temperature, the probability of desorption increases [14]. Therefore, the slow recovery ( $\sim$ minutes) for $1 \% \mathrm{H}_{2}$ at room temperature is expected.

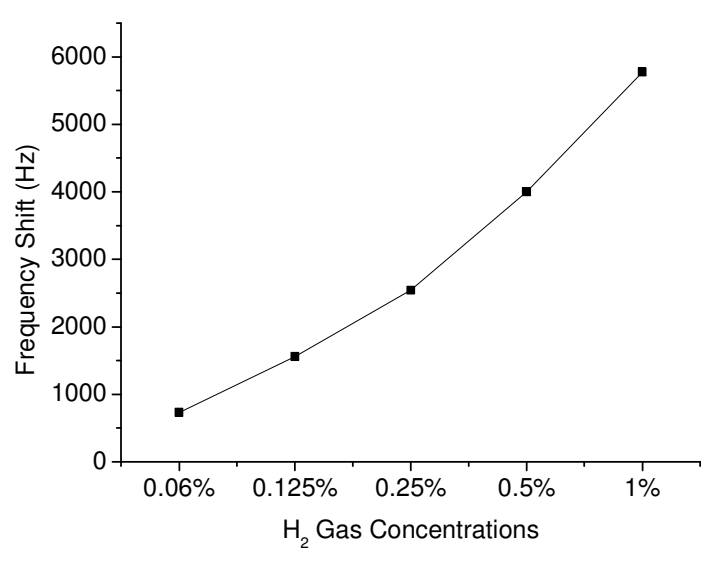

Figure 5: Frequency shifts for graphene-like nano-sheets/SAW sensors at room temperature

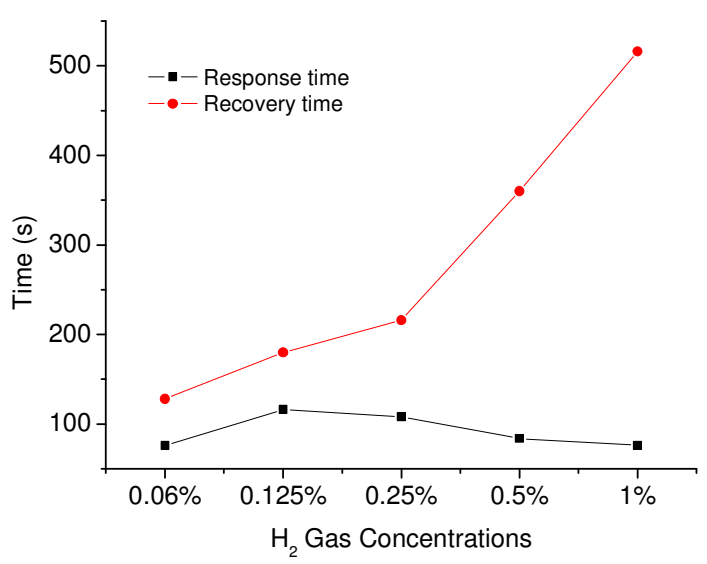

Figure 6: Response and recovery time vs $\mathrm{H}_{2}$ gas concentrations

\section{CONCLUSION}

We have successfully fabricated graphene-like nanosheets/ $\mathrm{LiTaO}_{3}$ SAW sensors and tested its sensing performance towards $\mathrm{H}_{2}$ gas. The combination of graphenelike nano-sheets and SAW device technology has promising environmental applications, owing to its room temperature operation. Such devices may find applications for low temperature $\mathrm{H}_{2}$ sensors, as our experiments have demonstrated that the graphene/SAW device shows good sensitivity towards $\mathrm{H}_{2}$ gas. 


\section{ACKNOWLEDGMENT}

Authors acknowledge Associate Prof. Dr. Johan du Plesis for help acquiring XPS spectrum.

\section{REFERENCES}

[1] L. C. Cadwallader and J. S. Herring, "Safety Issues with Hydrogen as a Vehicle Fuel," Idaho National Engineering and Environmental Laboratory, Lockheed Martin Idaho Technologies Company, Idaho Sept 1999.

[2] M. Heisel, "Industrial gases in the chemical industry." Linde Gas Pte Ltd., 2008

[3] G. W. Hunter et al., "Development of chemical sensor arrays for harsh environments and aerospace applications," in Sensors, Proceedings of IEEE, 2002, pp. 1126- 1133.

[4] K. S. Novoselov et al., "Electric field effect in atomically thin carbon films," 2004.

[5] C. Srinivasan, "Graphene - Mother of all graphitic materials," Current Science, vol. 92, pp. 1338-1339, 2007.

[6] F. Schedin, A. K. Geim, S. V. Morozov, E. W. Hill, P. Blake, M. I. Katsnelson, and K. S. Novoselov, "Detection of individual gas molecules adsorbed on graphene," Nature Materials, vol. 6, pp. 652$655,2007$.
[7] P. Joshi, A. Gupta, P. C. Eklund, and S. A. Tadigadapa, "On the possibility of a graphene based chemical sensors," in The 14th International Conference on Solid-State Sensors, Actuators and Microsystems, Lyon ,France, 2007, pp. 2325-2328.

[8] K. S. Subrahmanyam, S. R. C. Vivekchand, A. Govindaraj, and C. N. R. Rao, "A study of graphenes prepared by different methods: characterization, properties and solubilization," Journal of Material Chemistry, vol. 18, 2008, pp. 1517-1523

[9] C. Berger et al., "Ultrathin epitaxial graphite: 2D electron gas properties abd a route toward graphene-based nanoelectronics," Journal of Physical Chemistry B, vol. 108, pp. 19912-19916, 2004.

[10] A. K. Geim and K. S. Novoselov, "The rise of graphene," Nature Materials, vol. 6, 2007, pp. 183-191

[11] S. Patchkovskii, J. S. Tse, S. N. Yurchenko, L. Zhechkov, T. Heine, and G. Seifert, "Graphene nanstructures as tunable storage media for molecular hydrogen," Proceedings of the National Academy of Sciences of the United States of America, vol. 102, 2005, pp. 1043910444

[12] S. Gilje, S. Han, M. Wang, K. L. Wang, and R. B. Kaner, "A Chemical Route to Graphene for Device Applications," Nano Letters, 2007.

[13] R. Arsat, M. Breedon, M. Shafiei, K. Kalantar-zadeh, S. Gilje, R. Kaner, and W. Wlodarski, "Graphene-like nano-sheets based $\mathrm{LiTaO}_{3}$ surface acoustic wave $\mathrm{NO}_{2}$ gas sensor," unpublished.

[14] G. Liang, J. Huot, S. Boily, and R. Schulz, "Hydrogen desorption kinetics of a mechanically milled $\mathrm{MgH} 2+5$ at. $\% \mathrm{~V}$ nanocomposite," Journal of Alloys and Compounds, vol. 305, 2000, pp. 239-245, 